OPEN ACCESS

Edited by:

Xinchun Jin,

Capital Medical University, China

Reviewed by:

Daqing Ma,

Imperial College London,

United Kingdom

Hongfei Zhang,

Southern Medical University, China

*Correspondence:

Xiaomin Wu

wuxiaomin@hmc.edu.cn

Shengmei Zhu

smzhu20088@zju.edu.cn

tThese authors share first authorship

Specialty section:

This article was submitted to

Cellular Neurophysiology,

a section of the journal

Frontiers in Cellular Neuroscience

Received: 06 May 2021 Accepted: 10 September 2021

Published: 12 October 2021

Citation:

Ding Z, Guo S, Luo L, Zheng Y, Gan S, Kang $X$, Wu X and Zhu S (2021) Emerging Roles of Microglia in Neuro-vascular Unit: Implications of Microglia-Neurons Interactions.

Front. Cell. Neurosci. 15:706025. doi: 10.3389/fncel.2021.706025

\section{Emerging Roles of Microglia in Neuro-vascular Unit: Implications of Microglia-Neurons Interactions}

\author{
Zhe Ding ${ }^{1 \dagger}$, Shaohui Guo ${ }^{1 \dagger}$, Lihui Luo ${ }^{1}$, Yueying Zheng ${ }^{1}$, Shuyuan Gan ${ }^{1}$, Xianhui Kang ${ }^{1}$, \\ Xiaomin $\mathrm{Wu}^{2 *}$ and Shengmei Zhu ${ }^{1 *}$ \\ ${ }^{1}$ Department of Anesthesiology, The First Affiliated Hospital, Zhejiang University School of Medicine, Hangzhou, China, \\ ${ }^{2}$ Department of Anesthesiology, Zhejiang Provincial People's Hospital, People's Hospital of Hangzhou Medical College, \\ Hangzhou, China
}

Microglia, which serve as the defensive interface of the nervous system, are activated in many neurological diseases. Their role as immune responding cells has been extensively studied in the past few years. Recent studies have demonstrated that neuronal feedback can be shaped by the molecular signals received and sent by microglia. Altered neuronal activity or synaptic plasticity leads to the release of various communication messages from neurons, which in turn exert effects on microglia. Research on microglia-neuron communication has thus expanded from focusing only on neurons to the neurovascular unit (NVU). This approach can be used to explore the potential mechanism of neurovascular coupling across sophisticated receptor systems and signaling cascades in health and disease. However, it remains unclear how microglia-neuron communication happens in the brain. Here, we discuss the functional contribution of microglia to synapses, neuroimmune communication, and neuronal activity. Moreover, the current state of knowledge of bidirectional control mechanisms regarding interactions between neurons and microglia are reviewed, with a focus on purinergic regulatory systems including ATP- $P_{2} R Y_{12} R$ signaling, ATP-adenosine- $A_{1} R s / A_{2 A} R s$, and the ATP-pannexin 1 hemichannel. This review aims to organize recent studies to highlight the multifunctional roles of microglia within the neural communication network in health and disease.

Keywords: microglia, microglia-neurons communication, synaptic plasticity, neuronal activity, NVU, ATP

\section{INTRODUCTION}

Research on central nervous system (CNS) disorders has largely concentrated on neurons; however, an increasing body of research suggests that a purely neurocentral focus is insufficient. All cell types in the brain-including neuronal, glial, and vascular components such as endothelia, pericytes, and vascular smooth muscular cells-should be examined in an integrated context (Muoio et al., 2014). Together, these components are termed the neurovascular unit (NVU; Figure 1), which plays an important role in brain function and disease through cell-cell signaling (Iadecola, 2017). The NVU is reported to control blood-brain barrier (BBB) permeability and cerebral blood flow (CBF) and to regulate the neuronal 'milieu' (Zlokovic, 2011). Signaling perturbations within the NVU comprise potential mechanisms for neuronal dysfunction and injury (Sweeney et al., 2016; Yu et al., 2020). Recent studies have shown that the NVU contributes to both stroke and neurodegenerative diseases (Cai et al., 2017; Giaume et al., 2021; Minhas et al., 2021). In light of such findings, increasing research efforts have focused on the NVU as a therapeutic target (Quaegebeur et al., 2011). 


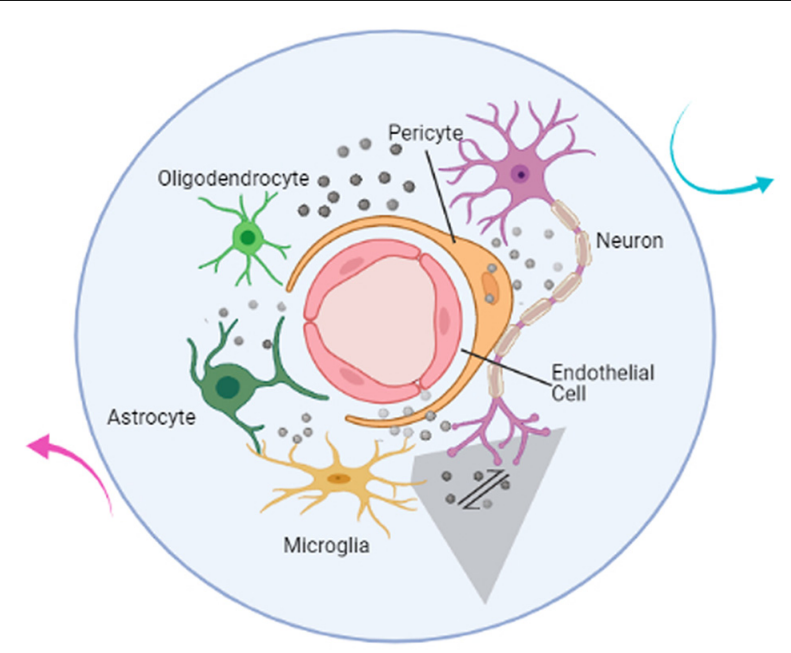

FIGURE 1 | |llustration of the components of the neurovascular unit. The neurovascular unit (NVU) is composed of neurons (purple), microglia (yellow), astrocytes (dark green), oligodendrocytes (light green), endothelial cells (red), and pericytes (orange). Based on intimate anatomical relationships and chemical signals (gray sphere) secreted by oneself or others, these components establish an anatomical and functional whole (blue circle) that participates in physiological and pathological processes in the brain. The communication network also includes the interactions between NVU and the outside world (cyan and magenta arrow). The mutual communication and cooperation between microglia and neurons (gray shaded trapezoid) in the brain is the focus of this article and will be discussed below.

Microglia perform an immune surveillance function through highly motile protrusions and ramified processes that sense their environment during their "resting" state or after activation in vivo (Nimmerjahn et al., 2005). Notably, microglia do not patrol in an aimless manner; rather, microglial surveillance is associated with neuronal activity and synaptic plasticity (Nayak et al., 2014). Emerging evidence suggests that microglia contribute to the development, maturation, and aging of the brain (Liu et al., 2020; Verkhratsky et al., 2021) and are recognized to express many risk genes for CNS disorders, including genes associated with Alzheimer's disease (AD), Parkinson's disease (PD), schizophrenia, autism, and multiple sclerosis (MS; Kékesi et al., 2019; Prinz et al., 2019). Neuronmicroglia signaling can be detected under physiological and pathological conditions in the brain (Cserép et al., 2020, 2021). The interactions between microglia and neurons establish complex regulatory loops that involve either the establishment of neural networks and maintenance of neural circuits in health or the development of neurological disorders in disease (Cserép et al., 2021).

Microglia, as a component of the NVU, play significant roles in neuroinflammation and innate immunity (Lehnardt, 2010; Joost et al., 2019). Not only do microglia-derived mediators participate in inflammation and immune-related responses they also serve as important messengers in cell-cell signaling between neuronal and glial cells. In this review, we discuss the evidence of the contribution of microglia to synapses, neuroimmune communication, and neuronal activity. In particular, we focus on bidirectional interactions between microglia and neurons that depend on soluble factors and intercellular signaling pathways, with the ultimate aim of better understanding the recently recognized functional roles of microglial actions in synaptic function, neuroimmune responses, and regulation of neural activity.

\section{A SYNAPTIC ROLE FOR MICROGLIA}

During postnatal development of the brain, microglia play major roles in the rapid elimination of dying neurons and synaptic structures (Tremblay et al., 2010; Ayata et al., 2018), synaptic pruning (Paolicelli et al., 2011; Schafer et al., 2012) and promoting the survival of cortical neurons (Ueno et al., 2013). Maternal immune activation (MIA), which is induced by injecting pregnant mice with polycytidylic acid (poly I:C), has been shown to have a significant impact on the pre-microglia (from embryonic day 14 to a few weeks after birth) of newborn offspring. As this impact on pre-microglia is influencing neurogenesis and synaptic pruning, it explains the later occurrence of behavioral disorders when the offspring are adults (Matcovitch-Natan et al., 2016). Emerging evidence suggests that microglia regulate activity-dependent structural plasticity and promote memory consolidation by locally clearing the extracellular matrix (ECM). Furthermore, cytokine interleukin-33 (IL-33), which is expressed by hippocampal neurons, significantly upregulates ECM protease genes (namely Adamts4 and $M m p 14$ ), thereby promoting microglial phagocytosis and engulfment of aggrecan around parvalbumin+ interneurons in the CA1 hippocampal subregion (Nguyen et al., 2020). Moreover, this mechanism is demonstrated in both young mouse ( 3 months) and old mouse (18 months) under physiological conditions.

The release of immunological mediators from microglia has also been shown to influence synaptic function. Beattie et al. (2002) reported that tumor-necrosis factor-alpha $(\mathrm{TNF} \alpha)$ significantly increases the mean miniature excitatory postsynaptic currents (mEPSCs) frequency and promotes the maintenance of synaptic strength, as indicated by mEPSCs at excitatory synapses that call for the continual presence of $\mathrm{TNF} \alpha$, which enhances synaptic efficacy by increasing surface expression of AMPA receptors. Since microglia are a source of $\mathrm{TNF} \alpha$, they potentiate glutamate-mediated neurotoxicity or participate in synaptic connectivity in an indirect way (Stellwagen and Malenka, 2006; Olmos and Lladó, 2014). Interleukin $1 \beta$ (IL-1 $\beta$ ) has been shown to impair long-term potentiation (LTP) directly at the synapse, which could explain why cognitive impairment is worse in aged hippocampal synapses (Prieto et al., 2015). Aberrant expression of IL- $1 \beta$ also results in damage to hippocampusdependent memory (Patterson, 2015). It is interesting that either TNF $\alpha$ or IL- $1 \beta$ plays a role in sleep regulation by changing neuromodulator/neurotransmitter receptor expression, resulting in altering neuron sensitivity (Krueger et al., 2011). Brainderived neurotrophic factor (BDNF), which is reported to influence synaptic plasticity, learning, and memory formation (Parkhurst et al., 2013; Leal et al., 2014), also appears to be a crucial signaling molecule in microglia-neuron communication. 
Preventing BDNF release from microglia has been shown to reverse allodynia and anion shift, and may thus provide a new therapeutic strategy for treating neuropathic pain (Coull et al., 2005). Interleukin 10 (IL-10), which acts on IL-10 receptors expressed on hippocampal neurons, plays a role in the induction of synaptic formation, including dendritic spines and excitatory and inhibitory synapses (Lim et al., 2013). It has been shown that IL-10 can facilitate LTP in the CA1 region of the hippocampus and increase synaptic strength, based on observations that the presynaptic fiber volley field excitatory postsynaptic potential (FV-fEPSP) slope increases after IL-10 treatment, thereby promoting synaptic plasticity (Nenov et al., 2019).

These soluble factors, which have been extensively studied in the context of neuroinflammation (Perry and Holmes, 2014), exert their effects on synaptic function as discussed above. The released cytokines act on neurons in a flexible way and are not limited by distance. Studies conducted in recent years have shown that the administration of cytokines, including $\mathrm{TNF} \alpha$ and IL-1 $\beta$, results in significantly increased astrogliosis at the brain injury site in neonatal mouse (Balasingam et al., 1994). Liddelow et al. (2017) reported that the release of IL-1 $\alpha$, TNF, and $\mathrm{Clq}$ from microglia is necessary and sufficient to induce a subtype of reactive astrocytes (termed A1 astrocytes), leading to impairment of neuronal survival, outgrowth, synaptogenesis, and phagocytosis and the death of neurons and oligodendrocytes. In response to cytokines, astrocytes can produce IL-1, IL-4, IL-6, IL-10, IL-12, TNF- $\alpha$, and interferon (IFN) to act on microglia (Benveniste, 1998). Taken together, these studies have shown that soluble factors contribute to the establishment of the interplay between different cell types within the NVU. The influence of microglia on neuronal networks appears to be sophisticated since interactions within the NVU cannot be ignored. Thus, the net effects must be taken into consideration. In light of this knowledge, the application should be carefully considered, since the ultimate effects of immunological mediators appear to depend on time, concentration, and environmental milieu.

\section{NEUROIMMUNE COMMUNICATION WITH MICROGLIA}

Microglia are the resident myeloid cells of the brain (Nayak et al., 2014). Equipped with specialized pattern recognition receptors (PRRs) that identify pathogen-associated molecular patterns (PAMPs) and host-derived danger-associated molecular patterns (DAMPs) in microorganisms, microglia play a key role in the neuroimmune system by quickly inducing a fine-tuned inflammatory response (Scheiblich et al., 2020). Activation of microglia that play a role in innate immune function is pivotal in neuroinflammation. In addition to their influences on synaptic plasticity, the release of cytokines and chemokines further amplifies the inflammatory process, which has been well documented in previous studies (Nayak et al., 2014; Prinz et al., 2019).

The nervous system is involved in regulating immunity and inflammation, whereas immune dysregulation and inflammation also affect brain function in disease (Pavlov et al., 2018). For instance, neural circuits may promote immunosuppression after injury or stroke by altering microglia function (Pavlov and Tracey, 2017). Notably, MIA, which involves alterations in the number and state of activated microglia, is closely associated with early disruptions in neurodevelopment and may result in later neuropsychiatric disorders in offspring, including anxiety-like or depression-like behavior, sensorimotor deficits, social deficits, and repetitive behaviors. Gumusoglu and Stevens (2019) proposed that the most common outcomes of maternal immune perturbation are elevations in the proinflammatory cytokines IL- 6 and IL-1 $\beta$, resulting in alterations in synapse formation and function and driving neural progenitors from a neurogenic to gliogenic fate. Brown et al. (2017) revealed that exposure to intrauterine inflammation altered metabolic profiles, including amino acid metabolism, purine metabolism, and lipid metabolism, in the amniotic fluid and fetal and neonatal brain of exposed offspring. Interestingly, the changes were sex-specific and persisted at a later time point $(48 \mathrm{~h}$ vs. $6 \mathrm{~h}$ after intrauterine inflammation; Brown et al., 2017). Furthermore, metabolic pathways disturbances, including mitochondrial function, arborization, and synapse formation, have been observed in the developmental interneurons of individuals with schizophrenia (SCZ) co-cultured with activated microglia. Intriguingly, SCZ cortical interneurons (cINs) show prolonged compromised metabolic pathways after removal of the activated microglia, which indicates an interaction between the genetic background of SCZ donors and the inflammatory environment provided by activated microglia (Park et al., 2020). It has been suggested that neuroinflammation mediated by microglia specifically acting on cINs has long-term effects during brain development. Notably, the microglial gene expression profiles of the offspring of mothers administered poly I:C were realigned with the normal microglial phenotype at adulthood, indicating that transient perturbation in the early stage of microglia development (such as the pre-microglia stage) may have far-reaching implications on the brain at adulthood (Matcovitch-Natan et al., 2016).

Recent studies suggest that any assessment of the impact of microglia-mediated immune responses on neurons should consider differences between short-term and long-term effects. It can be concluded that the activation of microglia following immune activation induces inflammation of the brain, which has a profound impact on neurodevelopment. Although microglia are primary initiators and effectors of neuroinflammation (Thurgur and Pinteaux, 2019), the pathological process includes the contributions of various cell types. It has been established that astrocytes along with microglia participate in the amplification of inflammation signals, which in turn may cause apoptosis of oligodendroglioma cancer cells (Liddelow et al., 2020). More precisely, microglia are essential for triggering the standard immune response of microglia with astrocytes, as confirmed by studies revealing that astrocytes show no response to pathogens/damage in the absence of microglia (Liu et al., 2021). At the same time, neuroimmune communication also occurs between microglia and endothelial cells, where microglia play a role in maintaining the integrity of the $\mathrm{BBB}$ and thus the influx of blood-derived molecules into the brain (Muoio et al., 2014). 
In addition, recent studies have uncovered that neuroimmune interactions are also important regulators of physiology (Huh and Veiga-Fernandes, 2020). For example, microglia work to maintain homeostasis in the brain, not only to resolve infections (Norris and Kipnis, 2019). Pavlov and Tracey (2017) revealed neural reflex regulation of immunity, finding that neural circuits are triggered by and control inflammation. Sensory neurons are capable of monitoring the immune state in the periphery and interacting with immune cells, which are defined as neuroimmune cell units. The communicating immune signals, which then activate the CNS, can derive from any major body organ including skin, lung, and intestines (reviewed in Huh and Veiga-Fernandes, 2020). Ultimately, the brain integrates neuroimmune communication in order to maintain a steady-state.

\section{MICROGLIA-NEURONS COMMUNICATION}

New evidence suggests that exacerbated activation of microglia can promote microglia-mediated neuronal degeneration (Zhao et al., 2018). It has been shown that loss of striatal microglia triggers seizures via activation of cortical and hippocampal neurons in mice (Badimon et al., 2020). Moreover, similar to inhibitory neurons, microglia can sense neuronal activation and then lower the activation of dopaminergic neurons to achieve negative feedback control of neuronal activity (Badimon et al., 2020). In addition, neuronal excitation affects the process extension and motility of microglia (Liu et al., 2019; Stowell et al., 2019). Overall, these studies support reciprocal connections between microglia and neurons. Understanding the molecular mechanisms that underlie these bidirectional interactions will be necessary to achieve an integrated view of microglia-neuron communication systems, thereby enabling real insight into the importance of these communication systems in the control of brain function.

\section{ATP- $\mathbf{P}_{2} R Y_{12}$ Signaling Is Essential for Microglia Neuron Communication}

Purinergic signaling plays a central role in microglia-neuron interactions, of which ATP is recognized as an efferent neurotransmitter in the CNS (Burnstock, 2006; Burnstock et al., 2011). ATP, which is mediated by G-protein-coupled $\mathrm{P}_{2}$ receptors, acts as an activity-dependent signal under physiological conditions or as a danger signal when dysfunction or damage occurs in the brain (Agostinho et al., 2020). There is evidence of the vesicular release of ATP from neurons (Pankratov et al., 2007; Larsson et al., 2012) and from astrocytes (Darby et al., 2003; Bowser and Khakh, 2007). ATP released by astrocytes regulates microglial branch dynamics in the intact brain and chemotactic responses of activated microglia toward the local injury site in the brain (Davalos et al., 2005). In neuron-microglia interactions, ATP supports communication from neurons to microglia via $\mathrm{P}_{2} \mathrm{RY}_{12}$ signaling activation (Badimon et al., 2020; Figure 2). In general, ATP results in a decrease in neuronal activity both in normal (Li et al., 2012) and in pathological conditions (Cserép et al., 2020). Microglial $\mathrm{P}_{2} \mathrm{RY}_{12}$ receptors are exclusively expressed by microglia and are viewed as an

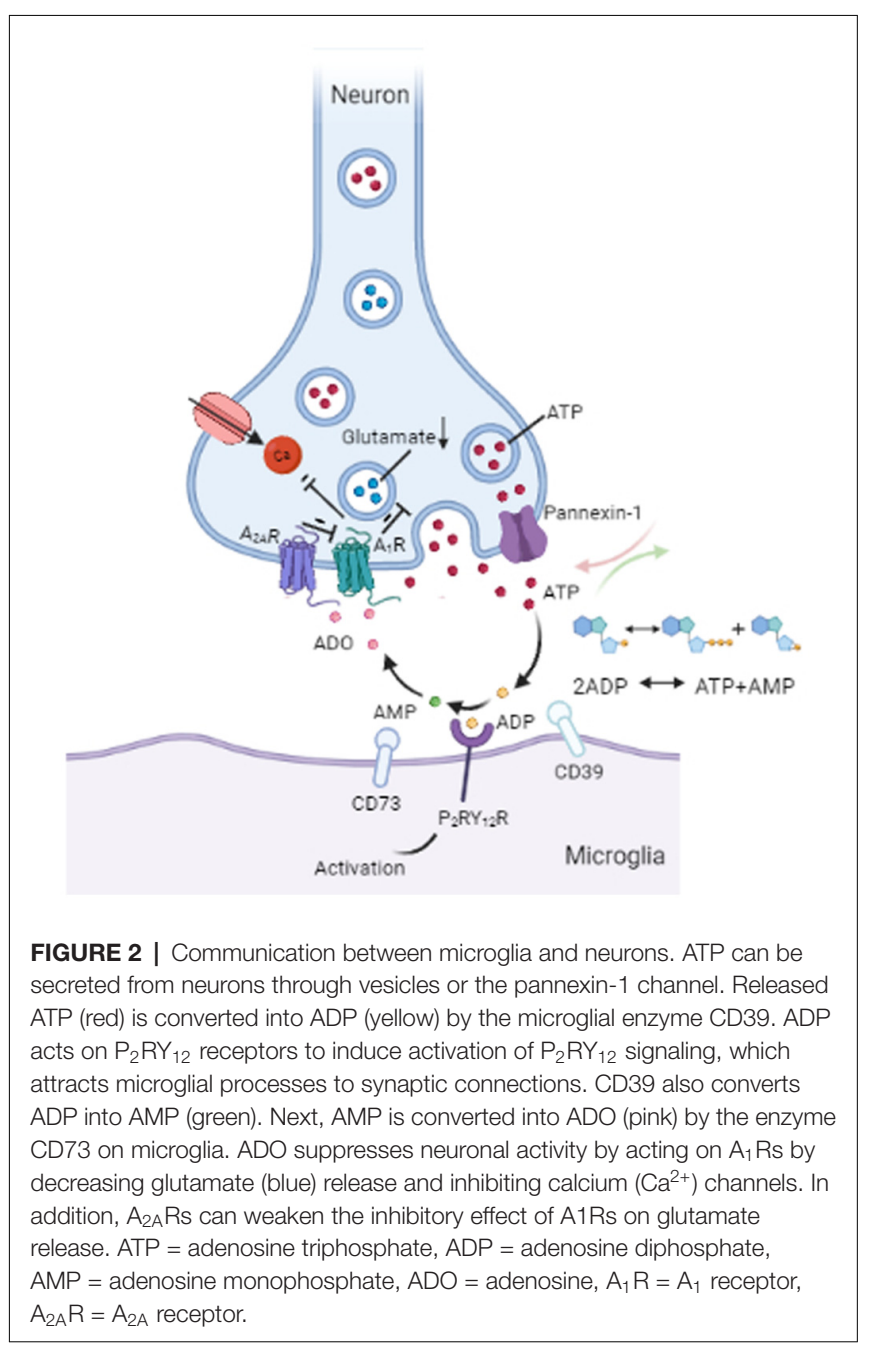

indispensable component of microglia-neuron junctions (Eyo et al., 2014; Cserép et al., 2020). 3D electron tomography showed that $\mathrm{P}_{2} \mathrm{RY}_{12}$ receptor density was negatively correlated with the distance between microglial and neuronal membranes within the junctions. Meanwhile, in vivo $2 \mathrm{P}$ imaging further confirmed that microglial process recruitment to somatic junctions is linked to the metabolic activity of neuronal mitochondria through a $\mathrm{P}_{2} \mathrm{RY}_{12}$ receptor-dependent mechanism (Cserép et al., 2020). Emerging evidence suggests that $\mathrm{P}_{2} \mathrm{RY}_{12} \mathrm{Rs}$ are involved in chemotaxis and the motility of microglia (Dissing-Olesen et al., 2014) as well as in microglia activation in the trigeminal nucleus caudalis (Jing et al., 2019), neuropathic pain (Tozaki-Saitoh et al., 2008; Gu et al., 2016), epilepticus (Avignone et al., 2008; Milior et al., 2020), and stroke (Kluge et al., 2017; Li et al., 2020). Thus, ATP- $\mathrm{P}_{2} \mathrm{RY}{ }_{12} \mathrm{R}$ signaling responses between microglia and neurons appear to contribute to an important loop in neural crosstalk (Figure 2).

\section{ATP-Adenosine- $A_{1} R s / A_{2 A} R s$ Are Essential for Microglia Neuron Communication}

Following ectoenzymatic breakdown of extracellular ATP, adenosine is produced and binds to $A_{1}$ or $A_{2 A}$ receptors in 
the brain, thereby regulating nerve activity and transmitter release (Fredholm et al., 2005). It has been demonstrated that adenosine (ADO) lowers neuronal excitability by acting on $A_{1}$ Rs and is a prominent physiological mediator of sleep homeostasis (Ribeiro et al., 2002). Recent studies have revealed that cholinergic and glutamatergic neurons show highly correlated activity with changes in adenosine concentration, with glutamatergic neurons contributing much more to adenosine increases in the mouse basal forebrain (BF; Peng et al., 2020). $A_{1}$ Rs and $A_{2 A}$ Rs are widely abundant in the brain, particularly in glutamatergic synapses (Yoon and Rothman, 1991; Rosin et al., 1998; Rebola et al., 2005). As a synaptic neuromodulator curtailing excitatory synaptic transmission, $\mathrm{A}_{1}$ Rs-mediated inhibition can fully block glutamatergic transmission (Coelho et al., 2006; Rodrigues et al., 2008) by inhibiting adenylate cyclase (AC), increasing potassium $\left(\mathrm{K}^{+}\right)$conductance, and inhibiting presynaptic calcium $\left(\mathrm{Ca}^{2+}\right)$ channels (Benarroch, 2008). $\mathrm{A}_{2 \mathrm{~A}} \mathrm{Rs}$ are activated by ATP-derived adenosine upon increased synaptic activity to act on synaptic plasticity (d'Alcantara et al., 2001; Augusto et al., 2013; Kerkhofs et al., 2018). In contrast, presynaptic $\mathrm{A}_{2 \mathrm{~A}} \mathrm{Rs}$ may form heteromeric receptor complexes with presynaptic $A_{1}$ Rs (Benarroch, 2008), which antagonizes the inhibitory effect of presynaptic $A_{1} R s$ on glutamate release from axon terminals in the striatum, cerebral cortex, and brainstem (Ribeiro et al., 2002; Boison, 2006). In addition to ATP- $\mathrm{P}_{2} \mathrm{RY}_{12} \mathrm{R}$ signaling as described above, a recent study demonstrated that ATP-adenosine- $\mathrm{A}_{1} \mathrm{Rs}$ signaling in mice suppresses D1 neuronal activity in the striatum, which could be regarded as a novel mechanism for communication between microglia and neurons (Badimon et al., 2020). Metabolic stress, such as ischemia, seizures, or trauma, may result in the upregulation of extracellular adenosine (Benarroch, 2008). The release of adenosine from parallel fibers has also been reported in the rat cerebellum. Moreover, activity-dependent adenosine release is known to regulate cerebellar circuit output through feedback inhibition of parallel fiber-Purkinje cell transmission (Wall and Dale, 2007). Much research consideration has surrounded adenosine as an endogenous neuromodulator in the CNS (Benarroch, 2008). Such studies have revealed roles for adenosine as well as adenosine receptors, greatly adding to our understanding while simultaneously expanding the complexity of the signaling system's mechanisms of action.

\section{ATP-Pannexin 1 Hemichannel Are Essential for Microglia Neuron Communication}

Pannexin 1 forms intercellular hemichannels and plays multiple roles in channel-mediated ATP release (Dahl and Locovei, 2006; Bhat and Sajjad, 2021). As nonjunctional membrane channels, pannexin 1 is abundantly expressed in the brain, including the hippocampus, olfactory bulb, cortex, and cerebellum (Bruzzone et al., 2003; Ray et al., 2005). These hemichannels can be activated by extracellular $\mathrm{Ca}^{2+}$ under physiological conditions (Barbe et al., 2006), and alterations in intracellular $\mathrm{Ca}^{2+}$ levels also open pannexin 1 channels (Giaume et al., 2021). Pannexin 1 channel-mediated ATP release has been shown to contribute to cell communication in vivo (Giaume et al., 2021; Figure 2). Reciprocal regulation between microglia and neurons involves pannexin-1 hemichannels in neurons and $\mathrm{ATP} / \mathrm{P}_{2}$ receptors in microglia, and so intraneuronal calcium plays a functional role in neuronal activity-induced microglia-neuron contact (Li et al., 2012). When pannexin 1 hemichannels are inhibited by trovafloxacin, both ATP release and migration of microglia are significantly repressed, resulting in the reduction of pro-inflammatory cytokines (Garg et al., 2018). Probenecid, which is an inhibitor of the pannexin 1 hemichannel, has been demonstrated to attenuate cognitive impairment after cecal ligation and puncture (CLP)-induced sepsis in mice by inhibiting pannexin 1-dependent ATP release in the hippocampus (Zhang et al., 2019). In addition, recent findings have proposed a role for pannexin-1 hemichannels in the suppression of glutamate release from hippocampal CA1 pyramidal neurons in male rat or mouse brains (Bialecki et al., 2020). In mice with pannexin-1 channels blocked or genetically deleted, the onset of seizures is reduced in neocortical slices in vitro (seizure-like events) and the hippocampal CA3 region in vivo, indicating a pivotal role of pannexin-1 in maintaining neuronal hyperexcitability (Aquilino et al., 2020). Similarly, the activation of pannexin-1 hemichannels in postoperative tissue samples from patients with epilepsy promotes seizure generation and maintenance through ATP signaling ex vivo (Dossi et al., 2018). Importantly, the ATP, glutamate, and other metabolites released from stimulated pannexin-1 hemichannels can go on participating in cell-to-cell communication in the brain (Aquilino et al., 2019).

Collectively, microglia are tightly regulated by the brain microenvironment and controlled by a sophisticated system of receptors and signaling cascades (Figure 2). However, there are some open questions worth discussing. For instance, ATP itself can act presynaptically, rather than solely postsynaptically, in the CNS (Cunha and Ribeiro, 2000). Despite a series of articles on the mechanisms of purinergic regulatory systems during microglia-neuron communication (Phillis and Wu, 1981), the balance between the effects of different signaling is yet to be considered. Besides acting on microglia and neurons, both ATP and adenosine are recognized to play a role in astrocyte proliferation and the formation of reactive astrocytes (Fumagalli et al., 2003). Similar to microglia, astrocytes are capable of releasing ATP and adenosine, which are then involved in cell-cell communication (Stout et al., 2002; Agostinho et al., 2020). ATP can be released along with neurotransmitters from nerve terminals, acting either as a neurotransmitter in central synapses or as a neuromodulator (Agostinho et al., 2020). Hence, the proposal of the NVU was quite significant and has inspired researchers to explore the signaling mechanisms spatially and temporally.

\section{CONCLUSIONS AND PERSPECTIVES}

In this review, we have brought together a series of articles exploring the roles of microglia, including their contributions to synapses, neuroimmune communication, and neuronal activity. 
In recent years, the development of imaging, genetics, and sequencing has made it possible to understand the real story of the complex and fascinating roles of microglia in health and disease. As a result, microglia manipulation has been proposed as a novel therapeutic method for modulating activity in various neurological diseases (Klawonn et al., 2021; Minhas et al., 2021). In the context of the neuroimmune roles of microglia, targeting microglia for immunotherapy in neurological disorders is aimed to maintain homeostasis of the brain by controlling neuroinflammation. However, the appropriate way to use microglia in immunotherapy remains unclear since they display both beneficial and detrimental roles. To gain deeper insight into the microglial shift from being protective to pathogenic, the molecular mechanisms involved warrant extensive study.

The overlapping results across MIA studies (reviewed in Gumusoglu and Stevens, 2019) inspire us that we can evaluate the psychiatric risk and disease etiologies by clinically testing the immune milieu of offspring. However, as discussed above, the transient perturbation in microglia development could have a profound impact on neurodevelopment. It still requires more efforts to prevent the occurrence of neuropsychiatric disorders in offspring. Moreover, inhibiting specific molecular pathways that mediate neuronal microglial communication is also a promising therapeutic approach, such as the application of probenecid in cerebral dysfunction of sepsis (Zhang et al., 2019). Novel research has reported that neuron-derived IL-33 (Nguyen et al., 2020) and astrocyte-derived IL-3 (McAlpine et al., 2021) as a key mediator of neuron-microglia crosstalk and astrocyte-microglia crosstalk, respectively, are associated

\section{REFERENCES}

Agostinho, P., Madeira, D., Dias, L., Simões, A. P., Cunha, R. A., and Canas, P. M. (2020). Purinergic signaling orchestrating neuron-glia communication. Pharmacol. Res. 162:105253. doi: 10.1016/j.phrs.2020.105253

Aquilino, M. S., Whyte-Fagundes, P., Lukewich, M. K., Zhang, L., Bardakjian, B. L., Zoidl, G. R., et al. (2020). Pannexin-1 deficiency decreases epileptic activity in mice. Int. J. Mol. Sci. 21:7510. doi: 10.3390/ijms21207510

Aquilino, M. S., Whyte-Fagundes, P., Zoidl, G., and Carlen, P. L. (2019). Pannexin1 channels in epilepsy. Neurosci. Lett. 695, 71-75. doi: 10.1016/j.neulet.2017. 09.004

Augusto, E., Matos, M., Sévigny, J., El-Tayeb, A., Bynoe, M. S., Müller, C. E., et al. (2013). Ecto-5'-nucleotidase (CD73)-mediated formation of adenosine is critical for the striatal adenosine A2A receptor functions. J. Neurosci. 33, 11390-11399. doi: 10.1523/JNEUROSCI.5817-12.2013

Avignone, E., Ulmann, L., Levavasseur, F., Rassendren, F., and Audinat, E. (2008). Status epilepticus induces a particular microglial activation state characterized by enhanced purinergic signaling. J. Neurosci. 28, 9133-9144. doi: 10.1523/JNEUROSCI.1820-08.2008

Ayata, P., Badimon, A., Strasburger, H. J., Duff, M. K., Montgomery, S. E., Loh, Y. E., et al. (2018). Epigenetic regulation of brain region-specific microglia clearance activity. Nat. Neurosci. 21, 1049-1060. doi: 10.1038/s41593-0180192-3

Badimon, A., Strasburger, H. J., Ayata, P., Chen, X., Nair, A., Ikegami, A., et al. (2020). Negative feedback control of neuronal activity by microglia. Nature 586, 417-423. doi: 10.1038/s41586-020-2777-8

Balasingam, V., Tejada-Berges, T., Wright, E., Bouckova, R., and Yong, V. W. (1994). Reactive astrogliosis in the neonatal mouse brain and its modulation by cytokines. J. Neurosci. 14, 846-856. doi: 10.1523/JNEUROSCI.14-02-00 846.1994 with memory consolidation. It provides a new strategy for the future treatments of age-related memory decline (IL33) and Alzheimer's disease (IL-3). In addition, eliminating about $80 \%$ microglia in the $5 x f A D$ mouse model of $\mathrm{AD}$ by blocking the CSF1 receptor is able to reverse deficits in contextual memory via preventing dendritic spine loss and neuronal loss, despite the disease being at a late stage (Spangenberg et al., 2016).

Another challenge is that bulk interventions using microglia could veil the true physical function of microglia since the overall outcome would be determined by microglia-mediated changes at different temporal and spatial scales (Cserép et al., 2021). The different effects could also be explained by the multifunctional roles of microglia and their communication within the NVU. Therefore, future studies should consider the molecular anatomy and functional heterogeneity of microglial processes and compartment-specific actions by microglia.

\section{AUTHOR CONTRIBUTIONS}

ZD and SG wrote this manuscript. LL, YZ, SG, and XK revised the manuscript. SZ and XW designed the general idea. All authors contributed to the article and approved the submitted version.

\section{FUNDING}

This study was supported by grants from the National Natural Science Foundation of China (Grant No. 81971008 and 81771194) to SZ and Zhejiang Basic Public Welfare Research Program (Grant No. LDG21H090006) to XW.

Barbe, M. T., Monyer, H., and Bruzzone, R. (2006). Cell-cell communication beyond connexins: the pannexin channels. Physiology 21, 103-114. doi: 10.1152/physiol.00048.2005

Beattie, E. C., Stellwagen, D., Morishita, W., Bresnahan, J. C., Ha, B. K., Von Zastrow, M., et al. (2002). Control of synaptic strength by glial TNF $\alpha$. Science 295, 2282-2285. doi: 10.1126/science. 1067859

Benarroch, E. E. (2008). Adenosine and its receptors: multiple modulatory functions and potential therapeutic targets for neurologic disease. Neurology 70, 231-236. doi: 10.1212/01.wnl.0000297939.18236.ec

Benveniste, E. N. (1998). Cytokine actions in the central nervous system. Cytokine Growth Factor Rev. 9, 259-275. doi: 10.1016/s1359-6101(98)00015-x

Bhat, E. A., and Sajjad, N. (2021). Human Pannexin 1 channel: insight in structurefunction mechanism and its potential physiological roles. Mol. Cell. Biochem. 476, 1529-1540. doi: 10.1007/s11010-020-04002-3

Bialecki, J., Werner, A., Weilinger, N. L., Tucker, C. M., Vecchiarelli, H. A., Egaña, J., et al. (2020). Suppression of presynaptic glutamate release by postsynaptic metabotropic NMDA receptor signalling to pannexin-1. J. Neurosci. 40, 729-742. doi: 10.1523/JNEUROSCI.0257-19.2019

Boison, D. (2006). Adenosine kinase, epilepsy and stroke: mechanisms and therapies. Trends Pharmacol. Sci. 27, 652-658. doi: 10.1016/j.tips.2006.10.008

Bowser, D. N., and Khakh, B. S. (2007). Vesicular ATP is the predominant cause of intercellular calcium waves in astrocytes. J. Gen. Physiol. 129, 485-491. doi: 10.1085/jgp.200709780

Brown, A. G., Tulina, N. M., Barila, G. O., Hester, M. S., and Elovitz, M. A. (2017) Exposure to intrauterine inflammation alters metabolomic profiles in the amniotic fluid, fetal and neonatal brain in the mouse. PLoS One 12:e186656. doi: 10.1371/journal.pone.0186656

Bruzzone, R., Hormuzdi, S. G., Barbe, M. T., Herb, A., and Monyer, H. (2003). Pannexins, a family of gap junction proteins expressed in brain. Proc. Natl. Acad. Sci. U S A 100, 13644-13649. doi: 10.1073/pnas.2233464100 
Burnstock, G. (2006). Purinergic signalling. Br. J. Pharmacol. 147, S172-S181. doi: 10.1038/sj.bjp.0706429

Burnstock, G., Krügel, U., Abbracchio, M. P., and Illes, P. (2011). Purinergic signalling: from normal behaviour to pathological brain function. Prog. Neurobiol. 95, 229-274. doi: 10.1016/j.pneurobio.2011.08.006

Cai, W., Zhang, K., Li, P., Zhu, L., Xu, J., Yang, B., et al. (2017). Dysfunction of the neurovascular unit in ischemic stroke and neurodegenerative diseases: an aging effect. Ageing Res. Rev. 34, 77-87. doi: 10.1016/j.arr.2016.09.006

Coelho, J. E., Rebola, N., Fragata, I., Ribeiro, J. A., de Mendonça, A., and Cunha, R. A. (2006). Hypoxia-induced desensitization and internalization of adenosine A1 receptors in the rat hippocampus. Neuroscience 138, 1195-1203. doi: 10.1016/j.neuroscience.2005.12.012

Coull, J. A., Beggs, S., Boudreau, D., Boivin, D., Tsuda, M., Inoue, K., et al. (2005). BDNF from microglia causes the shift in neuronal anion gradient underlying neuropathic pain. Nature 438, 1017-1021. doi: 10.1038/nature 04223

Cserép, C., Pósfai, B., and Dénes, Á. (2021). Shaping neuronal fate: functional heterogeneity of direct microglia-neuron interactions. Neuron 109, 222-240. doi: 10.1016/j.neuron.2020.11.007

Cserép, C., Pósfai, B., Lénárt, N., Fekete, R., László, Z. I., Lele, Z., et al. (2020). Microglia monitor and protect neuronal function through specialized somatic purinergic junctions. Science 367, 528-537. doi: 10.1126/science.aax6752

Cunha, R. A., and Ribeiro, J. A. (2000). ATP as a presynaptic modulator. Life Sci. 68, 119-137. doi: 10.1016/s0024-3205(00)00923-1

Dahl, G., and Locovei, S. (2006). Pannexin: to gap or not to gap, is that a question? IUBMB Life 58, 409-419. doi: 10.1080/15216540600794526

d'Alcantara, P., Ledent, C., Swillens, S., and Schiffmann, S. N. (2001). Inactivation of adenosine $\mathrm{A} 2 \mathrm{~A}$ receptor impairs long term potentiation in the accumbens nucleus without altering basal synaptic transmission. Neuroscience 107, 455-464. doi: 10.1016/s0306-4522(01)00372-4

Darby, M., Kuzmiski, J. B., Panenka, W., Feighan, D., and MacVicar, B. A. (2003). ATP released from astrocytes during swelling activates chloride channels. J. Neurophysiol. 89, 1870-1877. doi: 10.1152/jn.00510.2002

Davalos, D., Grutzendler, J., Yang, G., Kim, J. V., Zuo, Y., Jung, S., et al. (2005). ATP mediates rapid microglial response to local brain injury in vivo. Nat. Neurosci. 8, 752-758. doi: 10.1038/nn1472

Dissing-Olesen, L., LeDue, J. M., Rungta, R. L., Hefendehl, J. K., Choi, H. B., and MacVicar, B. A. (2014). Activation of neuronal NMDA receptors triggers transient ATP-mediated microglial process outgrowth. J. Neurosci. 34, 10511-10527. doi: 10.1523/JNEUROSCI.0405-14.2014

Dossi, E., Blauwblomme, T., Moulard, J., Chever, O., Vasile, F., Guinard, E., et al. (2018). Pannexin-1 channels contribute to seizure generation in human epileptic brain tissue and in a mouse model of epilepsy. Sci. Transl. Med. 10:eaar3796. doi: 10.1126/scitranslmed.aar3796

Eyo, U. B., Peng, J., Swiatkowski, P., Mukherjee, A., Bispo, A., and Wu, L. J. (2014). Neuronal hyperactivity recruits microglial processes via neuronal NMDA receptors and microglial P2Y12 receptors after status epilepticus. J. Neurosci. 34, 10528-10540. doi: 10.1523/JNEUROSCI.0416-14.2014

Fredholm, B. B., Chen, J. F., Cunha, R. A., Svenningsson, P., and Vaugeois, J. M. (2005). Adenosine and brain function. Int. Rev. Neurobiol. 63, 191-270. doi: 10.1016/S0074-7742(05)63007-3

Fumagalli, M., Brambilla, R., D’Ambrosi, N., Volonté, C., Matteoli, M., Verderio, C., et al. (2003). Nucleotide-mediated calcium signaling in rat cortical astrocytes: role of P2X and P2Y receptors. Glia 43, 218-230. doi: 10.1002/glia. 10248

Garg, C., Seo, J. H., Ramachandran, J., Loh, J. M., Calderon, F., and Contreras, J. E. (2018). Trovafloxacin attenuates neuroinflammation and improves outcome after traumatic brain injury in mice. J. Neuroinflammation 15:42. doi: 10.1186/s12974-018-1069-9

Giaume, C., Naus, C. C., Sáez, J. C., and Leybaert, L. (2021). Glial connexins and pannexins in the healthy and diseased brain. Physiol. Rev. 101, 93-145. doi: 10.1152/physrev.00043.2018

Gu, N., Eyo, U. B., Murugan, M., Peng, J., Matta, S., Dong, H., et al. (2016). Microglial P2Y12 receptors regulate microglial activation and surveillance during neuropathic pain. Brain Behav. Immun. 55, 82-92. doi: 10.1016/j.bbi. 2015.11.007

Gumusoglu, S. B., and Stevens, H. E. (2019). Maternal inflammation and neurodevelopmental programming: a review of preclinical outcomes and implications for translational psychiatry. Biol. Psychiatry 85, 107-121. doi: 10.1016/j.biopsych.2018.08.008

Huh, J. R., and Veiga-Fernandes, H. (2020). Neuroimmune circuits in inter-organ communication. Nat. Rev. Immunol. 20, 217-228. doi: 10.1038/s41577-0190247-z

Iadecola, C. (2017). The neurovascular unit coming of age: a journey through neurovascular coupling in health and disease. Neuron 96, 17-42. doi: 10.1016/j. neuron.2017.07.030

Jing, F., Zhang, Y., Long, T., He, W., Qin, G., Zhang, D., et al. (2019). $\mathrm{P} 2 \mathrm{Y} 12$ receptor mediates microglial activation via RhoA/ROCK pathway in the trigeminal nucleus caudalis in a mouse model of chronic migraine. J. Neuroinflammation 16:217. doi: 10.1186/s12974-019-1603-4

Joost, E., Jordão, M. J. C., Mages, B., Prinz, M., Bechmann, I., and Krueger, M. (2019). Microglia contribute to the glia limitans around arteries, capillaries and veins under physiological conditions, in a model of neuroinflammation and in human brain tissue. Brain Struct. Funct. 224, 1301-1314. doi: 10.1007/s00429019-01834-8

Kékesi, O., Liang, H., Münch, G., Morley, J. W., Gyengesi, E., and Buskila, Y. (2019). The differential impact of acute microglia activation on the excitability of cholinergic neurons in the mouse medial septum. Brain Struct. Funct. 224, 2297-2309. doi: 10.1007/s00429-019-01905-w

Kerkhofs, A., Canas, P. M., Timmerman, A. J., Heistek, T. S., Real, J. I., Xavier, C., et al. (2018). Adenosine $A_{2 A}$ receptors control glutamatergic synaptic plasticity in fast spiking interneurons of the prefrontal cortex. Front. Pharmacol. 9:133. doi: 10.3389/fphar.2018.00133

Klawonn, A. M., Fritz, M., Castany, S., Pignatelli, M., Canal, C., Similä, F., et al. (2021). Microglial activation elicits a negative affective state through prostaglandin-mediated modulation of striatal neurons. Immunity 54 , 225-234.e6. doi: 10.1016/j.immuni.2020.12.016

Kluge, M. G., Kracht, L., Abdolhoseini, M., Ong, L. K., Johnson, S. J., Nilsson, M., et al. (2017). Impaired microglia process dynamics post-stroke are specific to sites of secondary neurodegeneration. Glia 65, 1885-1899. doi: 10.1002/glia. 23201

Krueger, J. M., Clinton, J. M., Winters, B. D., Zielinski, M. R., Taishi, P., Jewett, K. A., et al. (2011). Involvement of cytokines in slow wave sleep. Prog. Brain Res. 193, 39-47. doi: 10.1016/B978-0-444-53839-0.00003-X

Larsson, M., Sawada, K., Morland, C., Hiasa, M., Ormel, L., Moriyama, Y., et al. (2012). Functional and anatomical identification of a vesicular transporter mediating neuronal ATP release. Cereb. Cortex 22, 1203-1214. doi: $10.1093 /$ cercor/bhr203

Leal, G., Comprido, D., and Duarte, C. B. (2014). BDNF-induced local protein synthesis and synaptic plasticity. Neuropharmacology 76, 639-656. doi: 10.1016/j.neuropharm.2013.04.005

Lehnardt, S. (2010). Innate immunity and neuroinflammation in the CNS: the role of microglia in Toll-like receptor-mediated neuronal injury. Glia 58, 253-263. doi: 10.1002/glia.20928

Li, Y., Du, X.-F., Liu, C.-S., Wen, Z.-L., and Du, J.-L. (2012). Reciprocal regulation between resting microglial dynamics and neuronal activity in vivo. Dev. Cell 23, 1189-1202. doi: 10.1016/j.devcel.2012.10.027

Li, F., Xu, D., Hou, K., Gou, X., and Li, Y. (2020). The role of P2Y12 receptor inhibition in ischemic stroke on microglia, platelets and vascular smooth muscle cells. J. Thromb. Thrombolysis 50, 874-885. doi: 10.1007/s11239-02002098-4

Liddelow, S. A., Guttenplan, K. A., Clarke, L. E., Bennett, F. C., Bohlen, C. J., Schirmer, L., et al. (2017). Neurotoxic reactive astrocytes are induced by activated microglia. Nature 541, 481-487. doi: 10.1038/nature 21029

Liddelow, S. A., Marsh, S. E., and Stevens, B. (2020). Microglia and astrocytes in disease: dynamic duo or partners in crime? Trends Immunol. 41, 820-835. doi: 10.1016/j.it.2020.07.006

Lim, S.-H., Park, E., You, B., Jung, Y., Park, A.-R., Park, S. G., et al. (2013). Neuronal synapse formation induced by microglia and interleukin 10. PLoS One 8:e81218. doi: 10.1371/journal.pone.0081218

Liu, L.-R., Liu, J.-C., Bao, J.-S., Bai, Q.-Q., and Wang, G.-Q. (2020). Interaction of microglia and astrocytes in the neurovascular unit. Front. Immunol. 11:1024. doi: 10.3389/fimmu.2020.01024

Liu, Y. J., Spangenberg, E., Tang, B., Holmes, T. C., Green, K. N., and Xu, X. (2021). Microglia elimination increases neural circuit connectivity and activity in adult 
mouse cortex. J. Neurosci. 41, 1274-1287. doi: 10.1523/JNEUROSCI.2140-20. 2020

Liu, Y. U., Ying, Y., Li, Y., Eyo, U. B., Chen, T., Zheng, J., et al. (2019). Neuronal network activity controls microglial process surveillance in awake mice via norepinephrine signaling. Nat. Neurosci. 22, 1771-1781. doi: 10.1038/s41593019-0511-3

Matcovitch-Natan, O., Winter, D. R., Giladi, A., Vargas Aguilar, S., Spinrad, A., Sarrazin, S., et al. (2016). Microglia development follows a stepwise program to regulate brain homeostasis. Science 353:aad8670. doi: 10.1126/science.aad8670

McAlpine, C. S., Park, J., Griciuc, A., Kim, E., Choi, S. H., Iwamoto, Y., et al. (2021). Astrocytic interleukin-3 programs microglia and limits Alzheimer's disease. Nature 595, 701-706. doi: 10.1038/s41586-021-03734-6

Milior, G., Morin-Brureau, M., Chali, F., Le Duigou, C., Savary, E., Huberfeld, G., et al. (2020). Distinct P2Y receptors mediate extension and retraction of microglial processes in epileptic and peritumoral human tissue. J. Neurosci. 40, 1373-1388. doi: 10.1523/JNEUROSCI.0218-19.2019

Minhas, P. S., Latif-Hernandez, A., McReynolds, M. R., Durairaj, A. S., Wang, Q., Rubin, A., et al. (2021). Restoring metabolism of myeloid cells reverses cognitive decline in ageing. Nature 590, 122-128. doi: 10.1038/s41586-02003160-0

Muoio, V., Persson, P. B., and Sendeski, M. M. (2014). The neurovascular unit-concept review. Acta Physiol. 210, 790-798. doi: 10.1111/apha.12250

Nayak, D., Roth, T. L., and McGavern, D. B. (2014). Microglia development and function. Annu. Rev. Immunol. 32, 367-402. doi: 10.1146/annurev-immunol032713-120240

Nenov, M. N., Malkov, A. E., Konakov, M. V., and Levin, S. G. (2019). Interleukin10 and transforming growth factor- $\beta 1$ facilitate long-term potentiation in CA1 region of hippocampus. Biochem. Biophys. Res. Commun. 518, 486-491. doi: 10.1016/j.bbrc.2019.08.072

Nguyen, P. T., Dorman, L. C., Pan, S., Vainchtein, I. D., Han, R. T., NakaoInoue, H., et al. (2020). Microglial remodeling of the extracellular matrix promotes synapse plasticity. Cell 182, 388-403.e15. doi: 10.1016/j.cell.2020.05. 050

Nimmerjahn, A., Kirchhoff, F., and Helmchen, F. (2005). Resting microglial cells are highly dynamic surveillants of brain parenchyma in vivo. Science 308, 1314-1318. doi: 10.1126/science. 1110647

Norris, G. T., and Kipnis, J. (2019). Immune cells and CNS physiology: microglia and beyond. J. Exp. Med. 216, 60-70. doi: 10.1084/jem.20180199

Olmos, G., and Lladó, J. (2014). Tumor necrosis factor $\alpha$ : a link between neuroinflammation and excitotoxicity. Mediators Inflamm. 2014:861231. doi: 10.1155/2014/861231

Pankratov, Y., Lalo, U., Verkhratsky, A., and North, R. A. (2007). Quantal release of ATP in mouse cortex. J. Gen. Physiol. 129, 257-265. doi: 10.1085/jgp. 200609693

Paolicelli, R. C., Bolasco, G., Pagani, F., Maggi, L., Scianni, M., Panzanelli, P., et al. (2011). Synaptic pruning by microglia is necessary for normal brain development. Science 333, 1456-1458. doi: 10.1126/science.1202529

Park, G.-H., Noh, H., Shao, Z., Ni, P., Qin, Y., Liu, D., et al. (2020). Activated microglia cause metabolic disruptions in developmental cortical interneurons that persist in interneurons from individuals with schizophrenia. Nat. Neurosci. 23, 1352-1364. doi: 10.1038/s41593-020-00724-1

Parkhurst, C. N., Yang, G., Ninan, I., Savas, J. N., Yates, J. R. III., Lafaille, J. J., et al. (2013). Microglia promote learning-dependent synapse formation through brain-derived neurotrophic factor. Cell 155, 1596-1609. doi: 10.1016/j.cell. 2013.11.030

Patterson, S. L. (2015). Immune dysregulation and cognitive vulnerability in the aging brain: interactions of microglia, IL- $1 \beta$, BDNF and synaptic plasticity. Neuropharmacology 96, 11-18. doi: 10.1016/j.neuropharm.2014. 12.020

Pavlov, V. A., Chavan, S. S., and Tracey, K. J. (2018). Molecular and functional neuroscience in immunity. Annu. Rev. Immunol. 36, 783-812. doi: 10.1146/annurev-immunol-042617-053158

Pavlov, V. A., and Tracey, K. J. (2017). Neural regulation of immunity: molecular mechanisms and clinical translation. Nat. Neurosci. 20, 156-166. doi: 10.1038/nn.4477

Peng, W., Wu, Z., Song, K., Zhang, S., Li, Y., and Xu, M. (2020). Regulation of sleep homeostasis mediator adenosine by basal forebrain glutamatergic neurons. Science 369:eabb0556. doi: 10.1126/science.abb0556
Perry, V. H., and Holmes, C. (2014). Microglial priming in neurodegenerative disease. Nat. Rev. Neurol. 10, 217-224. doi: 10.1038/nrneurol.2014.38

Phillis, J. W., and Wu, P. H. (1981). The role of adenosine and its nucleotides in central synaptic transmission. Prog. Neurobiol. 16, 187-239. doi: 10.1016/03010082(81)90014-9

Prieto, G. A., Snigdha, S., Baglietto-Vargas, D., Smith, E. D., Berchtold, N. C., Tong, L., et al. (2015). Synapse-specific IL-1 receptor subunit reconfiguration augments vulnerability to IL-1 $\beta$ in the aged hippocampus. Proc. Natl. Acad. Sci. US A 112, E5078-E5087. doi: 10.1073/pnas.1514486112

Prinz, M., Jung, S., and Priller, J. (2019). Microglia biology: one century of evolving concepts. Cell 179, 292-311. doi: 10.1016/j.cell.2019.08.053

Quaegebeur, A., Lange, C., and Carmeliet, P. (2011). The neurovascular link in health and disease: molecular mechanisms and therapeutic implications. Neuron 71, 406-424. doi: 10.1016/j.neuron.2011.07.013

Ray, A., Zoidl, G., Weickert, S., Wahle, P., and Dermietzel, R. (2005). Site-specific and developmental expression of pannexin 1 in the mouse nervous system. Eur. J. Neurosci. 21, 3277-3290. doi: 10.1111/j.1460-9568.2005.04139.x

Rebola, N., Rodrigues, R. J., Lopes, L. V., Richardson, P. J., Oliveira, C. R., and Cunha, R. A. (2005). Adenosine A1 and A2A receptors are co-expressed in pyramidal neurons and co-localized in glutamatergic nerve terminals of the rat hippocampus. Neuroscience 133, 79-83. doi: 10.1016/j.neuroscience.2005. 01.054

Ribeiro, J. A., Sebastião, A. M., and de Mendonça, A. (2002). Adenosine receptors in the nervous system: pathophysiological implications. Prog. Neurobiol. 68, 377-392. doi: 10.1016/s0301-0082(02)00155-7

Rodrigues, R. J., Canas, P. M., Lopes, L. V., Oliveira, C. R., and Cunha, R. A. (2008). Modification of adenosine modulation of acetylcholine release in the hippocampus of aged rats. Neurobiol. Aging 29, 1597-1601. doi: 10.1016/j. neurobiolaging.2007.03.025

Rosin, D. L., Robeva, A., Woodard, R. L., Guyenet, P. G., and Linden, J. (1998). Immunohistochemical localization of adenosine A2A receptors in the rat central nervous system. J. Comp. Neurol. 401, 163-186. doi: 10.1002/(SICI)1096-9861(19981116)401:2<163::AID-CNE2>3.0.CO;2-D

Schafer, D. P., Lehrman, E. K., Kautzman, A. G., Koyama, R., Mardinly, A. R., Yamasaki, R., et al. (2012). Microglia sculpt postnatal neural circuits in an activity and complement-dependent manner. Neuron 74, 691-705. doi: 10.1016/j.neuron.2012.03.026

Scheiblich, H., Trombly, M., Ramirez, A., and Heneka, M. T. (2020). Neuroimmune connections in aging and neurodegenerative diseases. Trends Immunol. 41, 300-312. doi: 10.1016/j.it.2020.02.002

Spangenberg, E. E., Lee, R. J., Najafi, A. R., Rice, R. A., Elmore, M. R., BlurtonJones, M., et al. (2016). Eliminating microglia in Alzheimer's mice prevents neuronal loss without modulating amyloid- $\beta$ pathology. Brain 139, 1265-1281. doi: 10.1093/brain/aww016

Stellwagen, D., and Malenka, R. C. (2006). Synaptic scaling mediated by glial TNF-

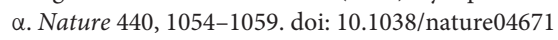

Stout, C. E., Costantin, J. L., Naus, C. C., and Charles, A. C. (2002). Intercellular calcium signaling in astrocytes via ATP release through connexin hemichannels. J. Biol. Chem. 277, 10482-10488. doi: 10.1074/jbc.M109902200

Stowell, R. D., Sipe, G. O., Dawes, R. P., Batchelor, H. N., Lordy, K. A., Whitelaw, B. S., et al. (2019). Noradrenergic signaling in the wakeful state inhibits microglial surveillance and synaptic plasticity in the mouse visual cortex. Nat. Neurosci. 22, 1782-1792. doi: 10.1038/s41593-019-0514-0

Sweeney, M. D., Ayyadurai, S., and Zlokovic, B. V. (2016). Pericytes of the neurovascular unit: key functions and signaling pathways. Nat. Neurosci. 19 , 771-783. doi: $10.1038 / \mathrm{nn} .4288$

Thurgur, H., and Pinteaux, E. (2019). Microglia in the neurovascular unit: bloodbrain barrier-microglia interactions after central nervous system disorders. Neuroscience 405, 55-67. doi: 10.1016/j.neuroscience.2018.06.046

Tozaki-Saitoh, H., Tsuda, M., Miyata, H., Ueda, K., Kohsaka, S., and Inoue, K. (2008). P2Y12 receptors in spinal microglia are required for neuropathic pain after peripheral nerve injury. J. Neurosci. 28, 4949-4956. doi: 10.1523/JNEUROSCI.0323-08.2008

Tremblay, M. Ė., Lowery, R. L., and Majewska, A. K. (2010). Microglial interactions with synapses are modulated by visual experience. PLoS Biol. 8:e1000527. doi: 10.1371/journal.pbio.1000527

Ueno, M., Fujita, Y., Tanaka, T., Nakamura, Y., Kikuta, J., Ishii, M., et al. (2013). Layer V cortical neurons require microglial support for survival 
during postnatal development. Nat. Neurosci. 16, 543-551. doi: 10.1038/ nn. 3358

Verkhratsky, A., Sun, D., and Tanaka, J. (2021). Snapshot of microglial physiological functions. Neurochem. Int. 144:104960. doi: 10.1016/j.neuint. 2021.104960

Wall, M. J., and Dale, N. (2007). Auto-inhibition of rat parallel fibre-Purkinje cell synapses by activity-dependent adenosine release. J. Physiol. 581, 553-565. doi: 10.1113/jphysiol.2006.126417

Yoon, K. W., and Rothman, S. M. (1991). Adenosine inhibits excitatory but not inhibitory synaptic transmission in the hippocampus. J. Neurosci. 11, 1375-1380. doi: 10.1523/JNEUROSCI.11-05-01375.1991

Yu, X., Ji, C., and Shao, A. (2020). Neurovascular unit dysfunction and neurodegenerative disorders. Front. Neurosci. 14:334. doi: 10.3389/fnins.2020. 00334

Zhang, Z., Lei, Y., Yan, C., Mei, X., Jiang, T., Ma, Z., et al. (2019). Probenecid relieves cerebral dysfunction of sepsis by inhibiting pannexin 1-dependent ATP release. Inflammation 42, 1082-1092. doi: 10.1007/s10753-019 $-00969-4$

Zhao, X., Liao, Y., Morgan, S., Mathur, R., Feustel, P., Mazurkiewicz, J., et al. (2018). Noninflammatory changes of microglia are sufficient to cause epilepsy. Cell Rep. 22, 2080-2093. doi: 10.1016/j.celrep.2018.02.004
Zlokovic, B. V. (2011). Neurovascular pathways to neurodegeneration in Alzheimer's disease and other disorders. Nat. Rev. Neurosci. 12, 723-738. doi: $10.1038 / \mathrm{nrn} 3114$

Conflict of Interest: The authors declare that the research was conducted in the absence of any commercial or financial relationships that could be construed as a potential conflict of interest.

Publisher's Note: All claims expressed in this article are solely those of the authors and do not necessarily represent those of their affiliated organizations, or those of the publisher, the editors and the reviewers. Any product that may be evaluated in this article, or claim that may be made by its manufacturer, is not guaranteed or endorsed by the publisher.

Copyright (C) 2021 Ding, Guo, Luo, Zheng, Gan, Kang, Wu and Zhu. This is an open-access article distributed under the terms of the Creative Commons Attribution License (CC BY). The use, distribution or reproduction in other forums is permitted, provided the original author(s) and the copyright owner(s) are credited and that the original publication in this journal is cited, in accordance with accepted academic practice. No use, distribution or reproduction is permitted which does not comply with these terms. 